\title{
The linguistic intuitionistic fuzzy set TOPSIS method for linguistic multi-criteria decision makings
}

\author{
Yue Ou ${ }^{1}$, Liangzhong $\mathrm{Yi}^{2}, \mathrm{Bin}_{\mathrm{Zou}}{ }^{3}$, Zheng Pei ${ }^{3}$ \\ ${ }^{1}$ School of Mechanical Engineering, Xihua University, \\ Chengdu, Sichuan 610039, China. \\ 2 Sichuan Police College, Luzhou, Sichuan 646000, China. \\ ${ }^{3}$ Center for Radio Administration \& Technology Development, Xihua University, \\ Chengdu, Sichuan 610039, China. \\ E-mail:pqyz@263.net
}

Received 9 June 2017

Accepted 14 September 2017

\begin{abstract}
In the paper, we express uncertain assessments information in linguistic multi-criteria decision makings (LMCDMs) as linguistic intuitionistic fuzzy sets, i.e., the decision maker provides membership and nonmembership fuzzy linguistic terms to represent uncertain assessments information of alternatives in LMCDMs, and present Hamming distance between two linguistic intuitionistic fuzzy sets. Then we propose the linguistic intuitionistic fuzzy set TOPSIS method for LMCDMs, compared with the traditional TOPSIS method, we provide different the positive ideal solution, the negative ideal solution and the relative closeness degrees of alternatives, in addition, we design an algorithm to finish the linguistic intuitionistic fuzzy set TOPSIS method for LMCDMs. We utilize a LMCDM problem to illustrate the performance, usefulness and effectiveness of the linguistic intuitionistic fuzzy set TOPSIS method, and compare it with the hesitant fuzzy linguistic multi-criteria optimization and compromise solution (HFL-VIKOR) method, the symbolic aggregation-based method and the hesitant fuzzy linguistic TOPSIS (HFL-TOPSIS) method in the example, results show that the linguistic intuitionistic fuzzy set TOPSIS method is a useful and alternative method for LMCDMs.
\end{abstract}

Keywords: The TOPSIS method; The 2-tuple linguistic model; Hesitant fuzzy linguistic term set; Intuitionistic fuzzy sets; Linguistic multi-criteria decision makings.

\section{Introduction}

We always face tasks and activities in which it is necessary to use decision making processes in our daily lives. Generally, decision making is a cognitive process based on different mental and reasoning processes that lead to the choice of a suitable alternative from a set of possible alternatives in a decision situation $^{1-4}$. Despite the existence of different decision making processes in the literature composed of different phases, the TOPSIS method proposed in $^{5}$ is useful, important and widely studied multiple attribute group decision making method, formally, the TOPSIS method originates from the concept that the selected alternative should have the shortest distance from the positive ideal solution and the farthest from the negative ideal solution, it's decision making process can be expressed in the following five steps ${ }^{6}: 1$ ) normalization of decision matrix; 2) construction of weighted normalized decision matrix; 3 ) determination of positive and negative ideal solutions; 4) calculation of separation measures and relative close- 
ness; 5) ranking of alternatives. After then, many extended TOPSIS methods have been applied to multiple attribute decision makings ${ }^{7-13}$, such as, $\mathrm{Chen}^{14}$ proposed an extended TOPSIS method for multiple attribute decision makings by considering triangular fuzzy numbers and defining crisp Euclidean distance between two fuzzy numbers. Similarly, Ashtiani et al. ${ }^{15}$ extended the TOPSIS method to solve multiple attribute decision making problem with the intervalvalued fuzzy sets. He and Gong ${ }^{16}$ proposed a natural generalization of the TOPSIS method to solve multiple attribute decision making problem with intuitionistic fuzzy sets. Liu, et al. ${ }^{17}$ developed a new TOPSIS method for decision making problems with interval-valued intuitionistic fuzzy data. Yue ${ }^{18}$ developed a method for decision making problems with interval number and extended his method to intuitionistic fuzzy sets.

Because of the inherent complexity and uncertainty of the decision situation or the existence of multiple and conflicting objectives in decision making problems, human beings often use fuzzy linguistic values to express complex or uncertain information in decision making process, and decision makings with fuzzy linguistic information attract many researchers ${ }^{19-29}$, in which, the 2-tuple linguistic model ${ }^{30}$ is a useful and important tool for expressing and dealing with linguistic information, which provides a continuous fuzzy representation for linguistic values by the translation of the linguistic value obtained from the symbolic computation to the closest linguistic value in the initial linguistic value set. Formally, the 2-tuple linguistic model consists of modeling the linguistic information by means of a pair of elements, one element is a linguistic value similar to the fuzzy linguistic approach whose semantics is provided by a fuzzy membership function and the syntax chosen according to the choices offered by the fuzzy linguistic approach, another element is a numerical value, also called symbolic translation, that indicates the translation of the fuzzy membership function which represents the closest linguistic value if it does not match exactly the computed linguistic information. Up to now, many new symbolic representation models to improve different aspects of the 2-tuple linguistic model have been developed and many different realworld decision makings based on 2-tuple linguistic model have been applied, such as, $\mathrm{Xu}^{31}$ introduced the extended linguistic variable based on the concept of virtual linguistic values to improve the operational laws of symbolic operations. Wang and $\mathrm{Hao}^{32}$ proposed the linguistic proportional 2-tuple model to represent linguistic information that is a generalization and extension of the 2-tuple linguistic model, Guo et al. ${ }^{33}$ later extended the linguistic proportional 2-tuple model by using a third parameter to deal with incomplete linguistic preferences. Dong et al. ${ }^{34}$ presented the concept of numerical scale with the aim of completing the 2-tuple linguistic model and proportional 2-tuple models and making the elicitation of information more consistent in different decision situations. $\mathrm{Li}^{35}$ proposed an extended 2-tuple linguistic model that fuses the use of virtual linguistic values and 2-tuple linguistic values. Yang ${ }^{36}$ developed the counted linguistic variable for representing and aggregating linguistic information with the aim of providing better results and being easier to understand. Wei ${ }^{37}$ investigated the 2-tuple linguistic multiple attribute group decision making problems in which the information about attribute weights is partially known. Cables et al. ${ }^{38}$ proposed a decision making method in which the decision makers provided their assessment information to represent their qualitative preferences under 2-tuple linguistic environment. Moreover, many 2-tuple linguistic aggregation operators have been proposed in literatures ${ }^{39-44}$ to make the aggregation of linguistic information much more flexible, we refer $^{4}$ for more details about 2-tuple linguistic model and decision makings based on 2-tuple linguistic model.

Recently, inspired by intuitionistic fuzzy sets and 2-tuple linguistic values, Chen, et al. ${ }^{45}$ developed the concept of linguistic intuitionistic fuzzy numbers where membership and and nonmembership are represented as 2-tuple linguistic values. In order to process the multiple attribute decision making with linguistic intuitionistic fuzzy numbers, they provided the linguistic score index and linguistic accuracy index of number, analyzed the operation laws for linguistic intuitionistic fuzzy numbers and 
the related properties of the operation laws. Furthermore, they developed the linguistic intuitionistic fuzzy weighted averaging operator, linguistic intuitionistic fuzzy ordered weighted averaging operator and linguistic intuitionistic fuzzy hybrid averaging (LIFHA) operator, which can be utilized to aggregate the linguistic intuitionistic fuzzy information. Based on linguistic intuitionistic fuzzy numbers, this paper propose the linguistic intuitionistic fuzzy set TOPSIS method for linguistic multi-criteria decision makings, in which, we present Hamming distance between two linguistic intuitionistic fuzzy sets and analyze it's several properties, then we provide the positive ideal solution, the negative ideal solution and the relative closeness degree of alternatives to solve LMCDMs. In numerical example, we compare the linguistic intuitionistic fuzzy set TOPSIS method with the HFL-VIKOR method ${ }^{46}$, the symbolic aggregation-based method ${ }^{47}$ and the HFL-TOPSIS method ${ }^{48}$. The rest of this paper is structured as follows: In Section 2, we briefly review some basic concepts and linguistic intuitionistic fuzzy numbers. In Section 3, we define Hamming distance between two linguistic intuitionistic fuzzy sets and analyze their several properties. In Section 4 , we present the framework of the linguistic intuitionistic fuzzy set TOPSIS method, and provide an algorithm to deal with LMCDMs in linguistic intuitionistic fuzzy set environment. In Section 5, we utilize a LMCDM problem to illustrate the practicality of the linguistic intuitionistic fuzzy set TOPSIS method and comparison results. We conclude the paper in Section 6.

\section{Preliminaries}

In this section, we briefly review some basic concepts in 2-tuple linguistic model and linguistic intuitionistic fuzzy numbers.

The 2-tuple fuzzy linguistic representation model consists of a pair of elements ${ }^{30}$, which is explained as follows: 1) Let $s_{i} \in S=\left\{s_{0}, s_{1}, s_{2}, \ldots, s_{g}\right\}$ be a initial linguistic term set, whose semantics is provided by a fuzzy membership function on a universe of discourse, an ordered structure provided in the linguistic term set is a total order, i.e., for any $s_{i}, s_{j} \in S, s_{i} \leqslant s_{j}$ if and only if $i \leqslant j$, moreover, a negation operator is $N e g\left(s_{i}\right)=s_{j}$ such that $j=g-i$ $(g+1$ is the cardinality), the maximal and minimal operators are $\max \left\{s_{i}, s_{j}\right\}=s_{j}$ and $\min \left\{s_{i}, s_{j}\right\}=s_{i}$ if $\left.s_{i} \leqslant s_{j} ; 2\right) \alpha$ is a numerical value that indicates error when a fuzzy membership function is translated to the closest linguistic term, i.e.,

$\alpha= \begin{cases}{[-0.5,0.5),} & \text { if } s_{i} \in\left\{s_{1}, s_{2}, \ldots, s_{g-1}\right\}, \\ {[0,0.5),} & \text { if } s_{i}=s_{0}, \\ {[-0.5,0),} & \text { if } s_{i}=s_{g} .\end{cases}$

Accordingly, the 2-tuple linguistic value is a pair of elements noted as $\left(s_{i}, \alpha\right)$, which can be used to express the linguistic information on a universe of discourse, for example, a set of nine linguistic terms $S$ on $[0,1]$ is $S=\left\{s_{0}\right.$ (extremely poor), $s_{1}$ (very poor), $s_{2}$ (poor), $s_{3}$ (slightly poor), $s_{4}$ (fair), $s_{5}$ (slightly good), $s_{6}$ (good), $s_{7}$ (very good), $s_{8}$ (extremely good) $\}$ and their fuzzy sets on $[0,1]$ are graphically shown in Fig.1.

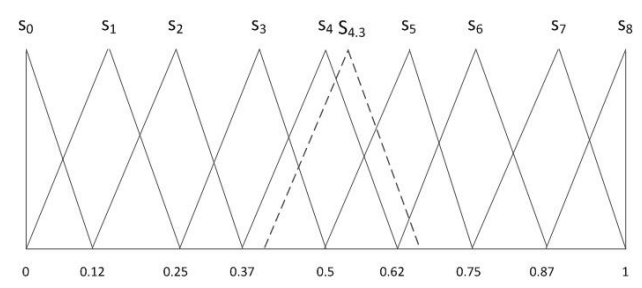

Fig. 1. Fuzzy sets of $S$ on $[0,1]$

In which, such as the 2-tuple linguistic value $\left(s_{4}, 0.3\right)=s_{4.3}$ corresponds to fuzzy set $s_{4.3}$ in Fig.1, formally, using the 2-tuple linguistic model can effectively avoid the loss and distortion of information in linguistic information processing, for simplify, we denote $S_{[0, g]}=\left\{s_{\alpha} \mid s_{0} \leqslant s_{\alpha} \leqslant s_{g}\right\}$ as all 2-tuple linguistic values on $[0,1]^{31}$.

Definition 1. ${ }^{45}$ Let $s_{\alpha}, s_{\beta} \in S_{[0, g]}$ and $\gamma=\left(s_{\alpha}, s_{\beta}\right)$, if $\alpha+\beta \leqslant g$, then we call $\gamma$ the linguistic intuitionistic fuzzy numbers defined on $S_{[0, g]}$. If $s_{\alpha}, s_{\beta} \in S$, then we call $\gamma$ the original linguistic intuitionistic fuzzy numbers, otherwise, we call $\gamma$ the virtual linguistic intuitionistic fuzzy numbers.

In the paper, we denote $\Gamma_{[0, g]}=\left\{\left(s_{\alpha}, s_{\beta}\right) \mid s_{\alpha}, s_{\beta} \in\right.$ $\left.S_{[0, g]}\right\}$ as all linguistic intuitionistic fuzzy numbers defined on $S_{[0, g]}$. For any $\left(s_{\alpha}, s_{\beta}\right),\left(s_{\alpha_{1}}, s_{\beta_{1}}\right)$, $\left(s_{\alpha_{2}}, s_{\beta_{2}}\right) \in \Gamma_{[0, g]}$, we have the following operators inspired by operations of intuitionistic fuzzy sets: 
- $\left(s_{\alpha_{1}}, s_{\beta_{1}}\right) \cup\left(s_{\alpha_{2}}, s_{\beta_{2}}\right)=\left(\max \left(s_{\alpha_{1}}, s_{\alpha_{2}}\right), \min \left(s_{\beta_{1}}, s_{\beta_{2}}\right)\right)$;

- $\left(s_{\alpha_{1}}, s_{\beta_{1}}\right) \cap\left(s_{\alpha_{2}}, s_{\beta_{2}}\right)=\left(\min \left(s_{\alpha_{1}}, s_{\alpha_{2}}\right), \max \left(s_{\beta_{1}}, s_{\beta_{2}}\right)\right)$;

- $\left(s_{\alpha}, s_{\beta}\right)^{c}=\left(s_{\beta}, s_{\alpha}\right)$;

- $\left(s_{\alpha_{1}}, s_{\beta_{1}}\right) \subseteq\left(s_{\alpha_{2}}, s_{\beta_{2}}\right)$ iff $s_{\alpha_{1}} \leqslant s_{\alpha_{2}}$, and $s_{\beta_{1}} \geqslant s_{\beta_{2}}$;

- $\left(s_{\alpha_{1}}, s_{\beta_{1}}\right)=\left(s_{\alpha_{2}}, s_{\beta_{2}}\right)$ iff $s_{\alpha_{1}}=s_{\alpha_{2}}$, and $s_{\beta_{1}}=s_{\beta_{2}}$.

Let $\left(s_{\alpha}, s_{\beta}\right),\left(s_{\alpha_{1}}, s_{\beta_{1}}\right),\left(s_{\alpha_{2}}, s_{\beta_{2}}\right) \in \Gamma_{[0, g]}, \lambda>0$, the following operations of linguistic intuitionistic fuzzy numbers have been defined ${ }^{45}$ :

- $\left(s_{\alpha_{1}}, s_{\beta_{1}}\right) \oplus\left(s_{\alpha_{2}}, s_{\beta_{2}}\right)=\left(s_{\alpha_{1}+\alpha_{2}-\frac{\alpha_{1} \alpha_{2}}{g}}, s_{\frac{\beta_{1} \beta_{2}}{g}}\right)$;

- $\left(s_{\alpha_{1}}, s_{\beta_{1}}\right) \otimes\left(s_{\alpha_{2}}, s_{\beta_{2}}\right)=\left(s \frac{\alpha_{1} \alpha_{2}}{g}, s_{\beta_{1}+\beta_{2}-\frac{\beta_{1} \beta_{2}}{g}}\right)$;

- $\lambda\left(s_{\alpha}, s_{\beta}\right)=\left(s_{g-g\left(1-\frac{\alpha}{g}\right)^{\lambda}}, s_{g\left(\frac{\beta}{g}\right)^{\lambda}}\right)$;

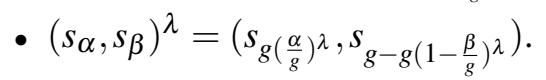

Accordingly, the linguistic intuitionistic fuzzy weighted averaging operator have been proposed: Let $\gamma_{j}=\left(s_{\alpha_{j}}, s_{\beta_{j}}\right) \in \Gamma_{[0, g]}(j=1, \ldots, n)$ and $w_{j}$ be the weight of $\gamma_{j}$, satisfying $0 \leqslant w_{j} \leqslant 1(j=1, \ldots, n)$ and $\sum_{j=1}^{n} w_{j}=1$. Then the linguistic intuitionistic fuzzy weighted averaging operator is

$$
\begin{aligned}
& \operatorname{LIFWA}\left(\gamma_{1}, \ldots, \gamma_{n}\right) \\
& =\left(s_{g-g \prod_{j=1}^{n}\left(1-\frac{\alpha_{j}}{g}\right)^{\omega_{j}}}, s_{g \prod_{j=1}^{n}\left(\frac{\beta_{j}}{g}\right)^{\omega_{j}}}\right) \text {. }
\end{aligned}
$$

As a special case, if $w=\left(\frac{1}{n}, \ldots, \frac{1}{n}\right)$, then Eq.(1) is reduced to the arithmetic aggregation operator:

$$
\begin{aligned}
& \operatorname{LIFWA}\left(\gamma_{1}, \ldots, \gamma_{n}\right) \\
= & \left(s_{g-g \prod_{j=1}^{n}\left(1-\frac{\alpha_{j}}{g}\right)^{\frac{1}{n}}}, s_{\left.g \prod_{j=1}^{n}\left(\frac{\beta_{j}}{g}\right)^{\frac{1}{n}}\right) .}\right.
\end{aligned}
$$

Let a LMCDM problem: The decision maker is asked to assess a set of alternatives $X=\left\{x_{1}, \ldots, x_{m}\right\}$ with respect to criteria $C=\left\{c_{1}, \cdots, c_{n}\right\}$, where the initial linguistic term set is $S=\left\{s_{0}, \cdots, s_{g}\right\}$, then the linguistic intuitionistic fuzzy assessment of alternative $x_{i}$ with respect to the criterion $c_{j}$ provided by the decision maker is represented as

$$
A_{j}=<x_{i}, s_{\alpha}^{j}\left(x_{i}\right), s_{\beta}^{j}\left(x_{i}\right)>,
$$

where $s_{\alpha}^{j}\left(x_{i}\right), s_{\beta}^{j}\left(x_{i}\right) \in S_{[0, g]}$ and $0 \leqslant \alpha+\beta \leqslant g$, $s_{\alpha}^{j}\left(x_{i}\right)$ represents the membership fuzzy linguistic assessment of $x_{i}$ provided by the decision maker with respect to the criterion $c_{j}, s_{\beta}^{j}\left(x_{i}\right)$ represents the nonmembership fuzzy linguistic assessment of $x_{i}$ provided by the decision maker with respect to the criterion $c_{j}$. Here, linguistic intuitionistic fuzzy assessments of alternatives are obviously linguistic intuitionistic fuzzy numbers on $S$.

For any $A_{j}$ of $x_{i}$, due to $\alpha+\beta \leqslant g$, we have $s_{\alpha}^{j}\left(x_{i}\right) \leqslant s_{g-\beta}^{j}\left(x_{i}\right)$, inspired by the intuitionistic fuzzy hesitation degree of intuitionistic fuzzy set, we call $s_{i} \in S$ as a linguistic intuitionistic fuzzy hesitation assessment of $x_{i}$ if $s_{\alpha}^{j}\left(x_{i}\right) \leqslant s_{i} \leqslant s_{g-\beta}^{j}\left(x_{i}\right)$, and all linguistic intuitionistic fuzzy hesitation assessments of $x_{i}$ are formed as a hesitant fuzzy linguistic term set ${ }^{47}: H_{S}^{j}\left(x_{i}\right)=\left\{s_{i} \in S \mid s_{\alpha}^{j}\left(x_{i}\right) \leqslant s_{i} \leqslant\right.$ $\left.s_{g-\beta}^{j}\left(x_{i}\right)\right\}$. In the following, we denote $A_{j}=<x_{i}$, $s_{\alpha}^{j}\left(x_{i}\right), s_{\beta}^{j}\left(x_{i}\right)>$ as $A_{j}=\left(s_{\alpha}, s_{\beta}\right)$ when $x_{i}$ is clear.

Example 1. Let alternatives $X=\left\{x_{1}, x_{2}, x_{3}\right\}$, criteria $C=\left\{c_{1}, c_{2}, c_{3}\right\}$ and the initial linguistic term set $S=\left\{s_{0}\right.$ (nothing), $s_{1}$ (very low), $s_{2}$ (low), $s_{3}$ (medium), $s_{4}$ (high), $s_{5}$ (very high), $s_{6}$ (perfect) $\}$, linguistic intuitionistic fuzzy assessments provided by the decision maker $d$ are shown in Table 1 , in which, such as for $\left(s_{1}, s_{3}\right)$ of $x_{1}$ with respect to $c_{1}$, the hesitant fuzzy linguistic term set is $H_{S}^{1}\left(x_{1}\right)=$ $\left\{s_{i} \in S \mid s_{1} \leqslant s_{i} \leqslant s_{6-3}\right\}=\left\{s_{1}, s_{2}, s_{3}\right\}$. All hesitant fuzzy linguistic term sets of three alternatives with respect to criteria are shown in Table 2.

Table 1. Linguistic intuitionistic assessments of alternatives.

\begin{tabular}{ccccc}
\hline & & $c_{1}$ & $c_{2}$ & $c_{3}$ \\
\hline & $x_{1}$ & $\left(s_{1}, s_{3}\right)$ & $\left(s_{4}, s_{1}\right)$ & $\left(s_{4}, s_{2}\right)$ \\
\hline$d$ & $x_{2}$ & $\left(s_{2}, s_{3}\right)$ & $\left(s_{3}, s_{3}\right)$ & $\left(s_{0}, s_{4}\right)$ \\
\hline & $x_{3}$ & $\left(s_{4}, s_{0}\right)$ & $\left(s_{1}, s_{4}\right)$ & $\left(s_{4}, s_{0}\right)$ \\
\hline
\end{tabular}

Table 2. The hesitant fuzzy linguistic term sets of alternatives based on Table 1.

\begin{tabular}{ccccc}
\hline & & $c_{1}$ & $c_{2}$ & $c_{3}$ \\
\hline & $x_{1}$ & $\left\{s_{1}, s_{2}, s_{3}\right\}$ & $\left\{s_{4}, s_{5}\right\}$ & $\left\{s_{4}\right\}$ \\
\hline$d$ & $x_{2}$ & $\left\{s_{2}, s_{3}\right\}$ & $\left\{s_{3}\right\}$ & $\left\{s_{0}, s_{1}, s_{2}\right\}$ \\
\hline & $x_{3}$ & $\left\{s_{4}, s_{5}, s_{6}\right\}$ & $\left\{s_{1}, s_{2}\right\}$ & $\left\{s_{4}, s_{5}, s_{6}\right\}$ \\
\hline
\end{tabular}




\section{The distance between two linguistic intuitionistic fuzzy numbers}

The distance measure is an important notion in existed TOPSIS methods. Inspired by the distance measure of intuitionistic fuzzy sets ${ }^{27}$, we can define the following Hamming distance between two linguistic intuitionistic fuzzy sets.

Definition 2. Let $A=\left(s_{\alpha_{1}}, s_{\beta_{1}}\right)$ and $B=\left(s_{\alpha_{2}}, s_{\beta_{2}}\right)$ be two linguistic intuitionistic fuzzy numbers. Then Hamming distance between $A$ and $B$ is as follows:

$$
d(A, B)=\frac{\left|\alpha_{1}-\alpha_{2}\right|+\left|\beta_{1}-\beta_{2}\right|+\left|\pi_{1}-\pi_{2}\right|}{2}
$$

where $\pi_{1}=g-\alpha_{1}-\beta_{1}, \pi_{2}=g-\alpha_{2}-\beta_{2}$.

Proposition 1. The distance $d(A, B)$ between $A$ and $B$ satisfies: (1) $0 \leqslant d(A, B) \leqslant g$; (2) $A=B$ iff $d(A, B)=0$; (3) $d(A, B)=d(B, A)$;(4) If $A \subseteq B \subseteq$ $C$ for $A, B, C \in \Gamma_{[0, g]}$, then $d(A, B) \leqslant d(A, C)$ and $d(B, C) \leqslant d(A, C)$.

Proof. (2) and (3) are obvious. We only prove (1) and (4).

According to $0 \leqslant \alpha_{i}, \beta_{i}, \pi_{i} \leqslant g$ and $\alpha_{i}+\beta_{i}+$ $\pi_{i}=g(i=1,2)$, we can obtain $0 \leqslant d(A, B)=$ $\frac{\left|\alpha_{1}-\alpha_{2}\right|+\left|\beta_{1}-\beta_{2}\right|+\left|\pi_{1}-\pi_{2}\right|}{2} \leqslant \frac{\alpha_{1}+\alpha_{2}+\beta_{1}+\beta_{2}+\pi_{1}+\pi_{2}}{2}=g$, i.e., (1) holds.

We have $\alpha_{1} \leqslant \alpha_{2} \leqslant \alpha_{3}$ and $\beta_{1} \geqslant \beta_{2} \geqslant \beta_{3}$ due to $A \subseteq B \subseteq C$. Then $d(A, C)-d(A, B)=$

$$
\begin{aligned}
& \frac{\left|\alpha_{1}-\alpha_{3}\right|+\left|\beta_{1}-\beta_{3}\right|+\left|\pi_{1}-\pi_{3}\right|}{2}-\frac{\left|\alpha_{1}-\alpha_{2}\right|+\left|\beta_{1}-\beta_{2}\right|+\left|\pi_{1}-\pi_{2}\right|}{2} \\
& =\frac{\alpha_{3}-\alpha_{1}+\beta_{1}-\beta_{3}+\left|\pi_{1}-\pi_{3}\right|}{2}-\frac{\alpha_{2}-\alpha_{1}+\beta_{1}-\beta_{2}+\left|\pi_{1}-\pi_{2}\right|}{2}= \\
& \frac{\alpha_{3}-\alpha_{2}+\beta_{2}-\beta_{3}+\left|\pi_{1}-\pi_{3}\right|-\left|\pi_{1}-\pi_{2}\right|}{2} \geqslant \frac{\alpha_{3}-\alpha_{2}+\beta_{2}-\beta_{3}-\left|\pi_{2}-\pi_{3}\right|}{2} \\
& =\frac{\alpha_{3}-\alpha_{2}+\beta_{2}-\beta_{3}-\left|\alpha_{3}-\alpha_{2}+\beta_{3}-\beta_{2}\right|}{2} \geqslant \\
& \frac{\alpha_{3}-\alpha_{2}+\beta_{2}-\beta_{3}-\left|\alpha_{3}-\alpha_{2}\right|-\left|\beta_{3}-\beta_{2}\right|}{2}=\frac{\alpha_{3}-\alpha_{2}+\beta_{2}-\beta_{3}-\alpha_{3}+\alpha_{2}-\beta_{2}+\beta_{3}}{2} \\
& =0, \text { i.e., } d(A, B) \leqslant d(A, C) \text { holds, similarly, } \\
& d(B, C) \leqslant d(A, C) \text { holds. }
\end{aligned}
$$

Example 2. In Example 1, for $A_{1}=\left(s_{1}, s_{3}\right)$ of $x_{1}$, $A_{1}=\left(s_{2}, s_{3}\right)$ of $x_{2}$ and $A_{3}=\left(s_{4}, s_{0}\right)$ of $x_{3}$, due to $s_{1}<s_{2}<s_{4}$ and $s_{3}=s_{3}>s_{0}$, we have $A_{1} \subseteq A_{2} \subseteq A_{3}$, in addition, $\pi_{1}=6-1-3=3, \pi_{2}=6-2-3=1$ and $\pi_{3}=6-4-0=2$,

$$
\begin{aligned}
& d\left(A_{1}, A_{2}\right)=\frac{|1-2|+|3-3|+|3-1|}{2}=1, \\
& d\left(A_{1}, A_{3}\right)=\frac{|1-4|+|3-0|+|3-2|}{2}=3.5, \\
& d\left(A_{2}, A_{3}\right)=\frac{|2-4|+|3-0|+|1-2|}{2}=3 .
\end{aligned}
$$

Hence, $d\left(A_{1}, A_{2}\right)<d\left(A_{1}, A_{3}\right)$ and $d\left(A_{2}, A_{3}\right)<$ $d\left(A_{1}, A_{3}\right)$.

\section{The linguistic intuitionistic fuzzy set TOPSIS method}

In this section, we present the linguistic intuitionistic fuzzy set TOPSIS method to solve LMCDMs, the method is mainly consisted of the four phases, i.e.,

1. construct the linguistic intuitionistic fuzzy decision matrix;

2. determine the positive and negative ideal solutions of alternatives;

3. calculate the relative closeness degree of every alternative;

4. rank alternatives according to their relative closenesses degree.

Where, we use the linguistic intuitionistic fuzzy decision matrix to express linguistic assessments of alternatives, i.e., in LMCDMs, we utilize linguistic intuitionistic fuzzy sets on $S$ and weights of criteria to represent uncertain linguistic assessments of alternatives provided by decision maker with respect to criteria, which is described in Subsection 4.1. In Subsection 4.2, based on the linguistic intuitionistic fuzzy decision matrix, we adopt $\cup$ and $\cap$ operations of linguistic intuitionistic fuzzy sets and the linguistic intuitionistic fuzzy weighted averaging operator to determine the positive and negative ideal solutions of alternatives. In Subsection 4.3, we use Hamming distance between linguistic intuitionistic fuzzy assessment of each alternative and the positive and negative ideal solutions to calculate the relative closeness degree of each alternative, then according to relative closeness degrees, we rank the preference 
order of all alternatives. We also provide Algorithm 1 in the end of Subsection 4.3 to automatically deal with LMCDM problems.

\subsection{The linguistic intuitionistic fuzzy decision matrix}

Let $W=\left\{w_{1}, \ldots, w_{n}\right\}$ be weights of criteria $C=$ $\left\{c_{1}, \cdots, c_{n}\right\}, A_{i j}=\left(s_{\alpha_{i j}}, s_{\beta_{i j}}\right)$ be linguistic intuitionistic fuzzy assessment of the alternative $x_{i}$ provided by decision maker with respect to the criterion $c_{j}$. Then the linguistic intuitionistic fuzzy decision matrix of a LMCDM can be formed as follows:

$$
\begin{aligned}
& D=\left(A_{i j}\right)_{m \times n}
\end{aligned}
$$

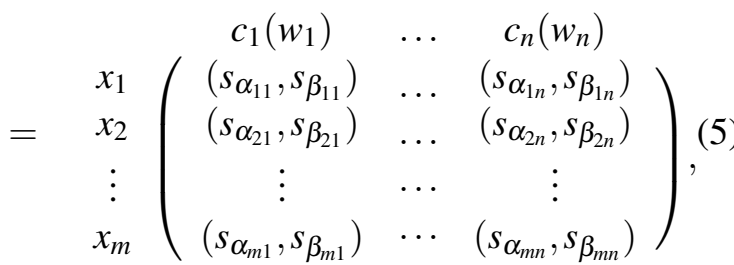

Example 3. In Example 1, suppose that weights of criteria $C=\left\{c_{1}, c_{2}, c_{3}\right\}$ is $W=\{0.3,0.4,0.3\}$, then the linguistic intuitionistic fuzzy decision matrix of the LMCDM is

$$
\begin{aligned}
& D=\left(A_{i j}\right)_{3 \times 3} \\
& =\begin{array}{lll}
c_{1}(0.3) & c_{2}(0.4) & c_{3}(0.3) \\
x_{1} & x_{2} \\
x_{3}
\end{array}\left(\begin{array}{lll}
\left(s_{1}, s_{3}\right) & \left(s_{4}, s_{1}\right) & \left(s_{4}, s_{2}\right) \\
\left(s_{2}, s_{3}\right) & \left(s_{3}, s_{3}\right) & \left(s_{0}, s_{4}\right) \\
\left(s_{4}, s_{0}\right) & \left(s_{1}, s_{4}\right) & \left(s_{4}, s_{0}\right)
\end{array}\right) .
\end{aligned}
$$

\subsection{The positive and negative ideal solutions of alternatives}

According to the linguistic intuitionistic fuzzy decision matrix $D$ (Eq.(5)), we use $\cup$ and $\cap$ operations of linguistic intuitionistic fuzzy numbers and the linguistic intuitionistic fuzzy weighted averaging operator to determine the positive and negative ideal solutions of alternatives, formally, for each column of $D, \cup$ and $\cap$ of linguistic intuitionistic fuzzy sets are as follows:

$$
\begin{aligned}
\vee c_{j} & =\left(s_{\vee \alpha_{j}}, s_{\vee \beta_{j}}\right)=\bigcup_{i=1}^{m} A_{i j}=\bigcup_{i=1}^{m}\left(s_{\alpha_{i j}}, s_{\beta_{i j}}\right) \\
& =\left(\max \left\{s_{\alpha_{1 j}}, \ldots, s_{\alpha_{m j}}\right\}, \min \left\{s_{\beta_{1 j}}, \ldots, s_{\beta_{m j}}\right\}\right), \\
\wedge c_{j} & =\left(s_{\wedge \alpha_{j}}, s_{\wedge \beta_{j}}\right)=\bigcap_{i=1}^{m} A_{i j}=\bigcap_{i=1}^{m}\left(s_{\alpha_{i j}}, s_{\beta_{i j}}\right) \\
& =\left(\min \left\{s_{\alpha_{1 j}}, \ldots, s_{\alpha_{m j}}\right\}, \max \left\{s_{\beta_{1 j}}, \ldots, s_{\beta_{m j}}\right\}\right) .
\end{aligned}
$$

Based on $\vee c_{j}, \wedge c_{j}$ and weights $W=\left\{w_{1}, \ldots, w_{n}\right\}$ of criteria $C=\left\{c_{1}, \cdots, c_{n}\right\}$, we utilize the linguistic intuitionistic fuzzy weighted averaging operator to determine the positive ideal solution (PIS) and the negative ideal solution (NIS) of alternatives, i.e.,

$$
\begin{aligned}
& \mathrm{PIS}=\left(s_{\alpha_{p}}, s_{\beta_{p}}\right)=\operatorname{LIFWA}\left(\vee c_{1}, \ldots, \vee c_{n}\right)
\end{aligned}
$$

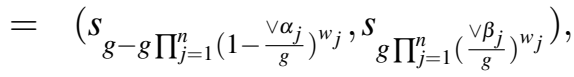

$$
\begin{aligned}
& \mathrm{NIS}=\left(s_{\alpha_{n}}, s_{\beta_{n}}\right)=\operatorname{LIFWA}\left(\wedge c_{1}, \ldots, \wedge c_{n}\right) \\
& =\left(s_{g-g \prod_{j=1}^{n}\left(1-\frac{\wedge \alpha_{j}}{g}\right)^{w_{j}}}, s_{g \prod_{j=1}^{n}\left(\frac{\wedge \beta_{j}}{g}\right)^{w_{j}}}\right) .
\end{aligned}
$$

Example 4. According to $D$ in Example 1, we have $\vee c_{1}=\bigcup_{i=1}^{3} A_{i 1}=\left(\max \left\{s_{1}, s_{2}, s_{4}\right\}, \min \{\right.$ $\left.\left.s_{3}, s_{3}, s_{0}\right\}\right)=\left(s_{4}, s_{0}\right), \wedge c_{1}=\bigcap_{i=1}^{3} A_{i 1}=\left(\min \left\{s_{1}, s_{2}\right.\right.$, $\left.\left.s_{4}\right\}, \max \left\{s_{3}, s_{3}, s_{0}\right\}\right)=\left(s_{1}, s_{3}\right), \vee c_{2}=\left(s_{4}, s_{1}\right), \wedge c_{2}=$ $\left(s_{1}, s_{4}\right), \vee c_{3}=\left(s_{4}, s_{0}\right)$ and $\wedge c_{2}=\left(s_{0}, s_{4}\right)$. Based on Eqs.(6) and (7), we can obtain PIS and NIS of $D$,

$$
\begin{aligned}
\mathrm{PIS} & =\operatorname{LIFWA}\left(\vee c_{1}, \vee c_{2}, \vee c_{3}\right) \\
& =\left(s_{6-6 \prod_{j=1}^{3}\left(1-\frac{\vee \alpha_{j}}{6}\right)^{w_{j}}}, s_{6 \prod_{j=1}^{3}\left(\frac{\vee \beta_{j}}{6}\right)^{w_{j}}}\right), \\
& =\left(s_{6-6 \times\left(1-\frac{4}{6}\right)}, s_{6 \times 0 \times \frac{1}{6} \times 0}\right) \\
& =\left(s_{4}, s_{0}\right), \\
\mathrm{NIS} & =\operatorname{LIFWA}\left(\wedge c_{1}, \wedge c_{2}, \wedge c_{3}\right) \\
& =\left(s_{6-6 \prod_{j=1}^{3}\left(1-\frac{\wedge \alpha_{j}}{6}\right)^{w_{j}}}, s_{6 \prod_{j=1}^{3}\left(\frac{\wedge \beta_{j}}{6}\right)^{w_{j}}}\right) \\
& =\left(s_{6-6 \times\left(1-\frac{1}{6}\right.}\right)^{0.7} \times 1 \\
& \left.s_{6 \times\left(\frac{3}{6}\right)^{0.3} \times\left(\frac{4}{6}\right)^{0.7}}\right) \\
& \doteq\left(s_{0.72}, s_{3.65}\right) .
\end{aligned}
$$

\subsection{The relative closeness and the ranking of alternatives}

To rank alternatives, we first calculate the relative closeness degree of every alternative, theoretically, 
the relative closeness degree is determined by distances between the linguistic intuitionistic fuzzy assessment of each alternative and the positive and negative ideal solutions. Based on Eq.(5), we use the linguistic intuitionistic fuzzy weighted averaging operator to obtain the linguistic intuitionistic fuzzy set of each alternative in $D$, i.e., for each row of $D$, the linguistic intuitionistic fuzzy set $A_{i}$ of $x_{i}$ is

$$
\begin{aligned}
A_{i} & =\left(s_{\alpha_{i}}, s_{\beta_{i}}\right)=\operatorname{LIFWA}\left(A_{i 1}, \ldots, A_{i n}\right) \\
& =\left(s_{g-g \prod_{j=1}^{n}\left(1-\frac{\alpha_{i j}}{g}\right)^{w_{j}}}, s_{g \prod_{j=1}^{n}\left(\frac{\beta_{i j}}{g}\right)^{w_{j}}}\right) .
\end{aligned}
$$

Based on $A_{i}$ of $x_{i}$, PIS (Eq.(6)), NIS (Eq.(7)) and Eq.(4), Hamming distances between the linguistic intuitionistic fuzzy set of each alternative and the positive and negative ideal solutions are

$$
\begin{aligned}
& d\left(A_{i}, \mathrm{PIS}\right)=\frac{\left|\alpha_{i}-\alpha_{p}\right|+\left|\beta_{i}-\beta_{p}\right|+\left|\pi_{i}-\pi_{p}\right|}{2}, \\
& d\left(A_{i}, \mathrm{NIS}\right)=\frac{\left|\alpha_{i}-\alpha_{n}\right|+\left|\beta_{i}-\beta_{n}\right|+\left|\pi_{i}-\pi_{n}\right|}{2},
\end{aligned}
$$

where, $\pi_{i}=g-\alpha_{i}-\beta_{i}, \pi_{p}=g-\alpha_{p}-\beta_{p}$ and $\pi_{n}=$ $g-\alpha_{n}-\beta_{n}$.

Originated from the TOPSIS method, the ranking of alternatives is based on "the shortest distance from the positive ideal solution and the farthest from the negative ideal solution", formally, this is also fulfilled by the relative closeness degree of each alternative in existed TOPSIS methods. Based on Hamming distance between the linguistic intuitionistic fuzzy set of each alternative and the positive and negative ideal solutions (Eqs.(9) and (10)), we provide the following relative closeness degree $C\left(x_{i}\right)$ of each alternative $x_{i}$,

$$
\begin{array}{r}
d_{\text {max }}^{-}=\max \left\{d\left(A_{1}, \mathrm{NIS}\right), \ldots, d\left(A_{m}, \mathrm{NIS}\right)\right\}, \\
d_{\text {min }}^{+}=\min \left\{d\left(A_{1}, \mathrm{PIS}\right), \ldots, d\left(A_{m}, \mathrm{PIS}\right)\right\}, \\
C\left(x_{i}\right)=\frac{1}{2}\left(\frac{d\left(A_{i}, \mathrm{NIS}\right)}{d_{\max }^{-}}+\frac{d_{\text {min }}^{+}}{d\left(A_{i}, \mathrm{PIS}\right)}\right) .
\end{array}
$$

Formally, $C\left(x_{i}\right)$ is in $[0,1]$ for any alternative $x_{i}$ and a monotone function in its components, i.e., $C\left(x_{i}\right)$ is increasing for $d\left(A_{i}\right.$, NIS $)$, and decreasing for $d\left(A_{i}\right.$, PIS $)$. Based on relative closeness degrees of alternatives, we can obtain the ranking of alternatives as follows: $\forall x_{i}, x_{i^{\prime}} \in X$,

$$
x_{i} \prec x_{i^{\prime}} \text { if and only if } C\left(x_{i}\right) \leqslant C\left(x_{i^{\prime}}\right) .
$$

Example 5. According to $D$ in Example 1 and Eq.(8), we obtain the linguistic intuitionistic fuzzy assessment of each alternative as follows:

$$
\begin{aligned}
& A_{1}=\operatorname{LIFWA}\left(\left(s_{1}, s_{3}\right),\left(s_{4}, s_{1}\right),\left(s_{4}, s_{2}\right)\right)
\end{aligned}
$$

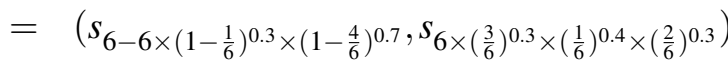

$$
\begin{aligned}
& \doteq\left(s_{3.38}, s_{1.71}\right) \text {, } \\
& A_{2}=\operatorname{LIFWA}\left(\left(s_{2}, s_{3}\right),\left(s_{3}, s_{3}\right),\left(s_{0}, s_{4}\right)\right) \\
& \doteq\left(s_{1.98}, s_{3.31}\right) \text {, } \\
& A_{3}=\operatorname{LIFWA}\left(\left(s_{4}, s_{0}\right),\left(s_{1}, s_{4}\right),\left(s_{4}, s_{0}\right)\right)
\end{aligned}
$$

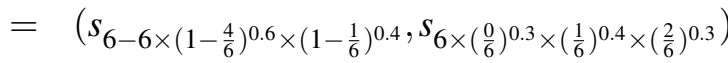

$$
\begin{aligned}
& \doteq\left(s_{3.10}, s_{0}\right) \text {, }
\end{aligned}
$$

Based on PIS and NIS in Example 4 and Eqs.(9) and (10), we can obtain $d\left(A_{1}\right.$, PIS $)=$ $\frac{|3.38-4|+|1.71-0|+|0.91-2|}{2}=1.71$ and $d\left(A_{1}\right.$, NIS $)=$ $\frac{|3.38-0.72|+|1.71-3.65|+|0.91-1.63|}{2}=2.16, \quad$ similarly, $d\left(A_{2}, \mathrm{PIS}\right)=3.31$ and $d\left(A_{2}, \mathrm{NIS}\right)=1.26$, $d\left(A_{3}, \mathrm{PIS}\right)=0.795$ and $d\left(A_{3}, \mathrm{NIS}\right)=3.545$. According to Eqs.(11), (12) and (13), we obtain

$$
\begin{aligned}
d_{\text {max }}^{-} & =\max \{2.16,1.26,3.545\}=3.545, \\
d_{\text {min }}^{+} & =\min \{1.71,3.31,0.795\}=0.795, \\
C\left(x_{1}\right) & =\frac{1}{2}\left(\frac{2.16}{3.545}+\frac{0.795}{1.71}\right)=0.535, \\
C\left(x_{2}\right) & =\frac{1}{2}\left(\frac{1.26}{3.545}+\frac{0.795}{3.31}\right)=0.30, \\
C\left(x_{3}\right) & =\frac{1}{2}\left(\frac{3.545}{3.545}+\frac{0.795}{0.795}\right)=1 .
\end{aligned}
$$

Hence, according to Eq.(14), the ranking of $\left\{x_{1}, x_{2}, x_{3}\right\}$ is $x_{2} \prec x_{1} \prec x_{3}$, i.e., $x_{3}$ is the most satisfying alternative.

Based on discussions in Subsections 4.1, 4.2 and 4.3 , we provide the following algorithm to carry out the linguistic intuitionistic fuzzy set TOPSIS method to solve LMCDMs.

\section{Algorithm 1}

Input: The numbers of alternatives $(m)$ and criteria (n), the linguistic term set $S=\left\{s_{0}, s_{1}, \cdots, s_{g}\right\}$.

Output: The ranking of alternatives and the most satisfying alternative.

Begin 
Step 1: According to the membership and nonmembership fuzzy linguistic assessments and Eq.(5), the linguistic intuitionistic fuzzy decision matrix $D$ of the LMCDM problem can be constructed;

Step 2: For each column of $D$, calculate $\vee c_{j}$ and $\wedge c_{j}$, then the linguistic intuitionistic fuzzy weighted averaging operator is used to determine PIS (Eq.(6)) and NIS (Eq.(7));

Step 3: For each row of $D$, the linguistic intuitionistic fuzzy weighted averaging operator is used to obtain the linguistic intuitionistic fuzzy assessment $A_{i}$ (Eq.(8)) of each alternative $x_{i}$;

Step 4: For each alternative, Hamming distances $d\left(A_{i}, \mathrm{PIS}\right)$ and $d\left(A_{i}, \mathrm{NIS}\right)$ between each $A_{i}$ and PIS (or NIS) are calculated by Eqs.(9) and (10), the maximum Hamming distance $d_{\max }^{-}$(Eq.(11)) of all $d\left(A_{i}, \mathrm{NIS}\right)(i=1, \ldots, m)$ and the minimum Hamming distance $d_{\text {min }}^{+}$(Eq.(12)) of all $d\left(A_{i}\right.$, PIS $)(i=$ $1, \ldots, m)$ are obtained, then the relative closeness degree $C\left(x_{i}\right)$ of each alternative $x_{i}$ is calculated by Eq.(13);

Output: $x_{i} \prec x_{i^{\prime}}$ if and only if $C\left(x_{i}\right) \leqslant C\left(x_{i^{\prime}}\right)$ by using Eq.(14).

end

\section{Numerical example}

In this section, we utilize an example to illustrate the practicality of the linguistic intuitionistic fuzzy set TOPSIS method, and compare the linguistic intuitionistic fuzzy set TOPSIS method with the HFLVIKOR method ${ }^{46}$, the symbolic aggregation-based method $^{47}$ and the HFL-TOPSIS method ${ }^{48}$. The example initially used in reference ${ }^{46}$ to show the HFLVIKOR method.

Example 6. ${ }^{46}$ A company intends to select an ERP system to implement from three candidates $A=$ $\left\{a_{1}, a_{2}, a_{3}\right\}$. To make a more reasonable decision, the chief information officer (CIO) of the company assesses the candidate ERP systems in terms of three criteria, i.e., $c_{1}$ (potential cost), $c_{2}$ (function), and $c_{3}$ (operation complexity). The weights of these criteria are $0.3,0.5$ and 0.2. Since the three criteria are qualitative, the $\mathrm{CIO}$ gives his assessment values in linguistic expressions (shown in Table 3). Reference ${ }^{46}$ used $S=\left\{\right.$ none $\left(s_{-3}\right)$, very low $\left(s_{-2}\right)$, low $\left(s_{-1}\right)$, medium $\left(s_{0}\right)$, high $\left(s_{1}\right)$, very high $\left(s_{2}\right)$, perfect $\left.\left(s_{2}\right)\right\}$. Here, we use the linguistic term set $S^{\prime}=\left\{\right.$ none $\left(s_{0}\right)$, very low $\left(s_{1}\right)$, low $\left(s_{2}\right)$, medium $\left(s_{3}\right)$, high $\left(s_{4}\right)$, very high $\left(s_{5}\right)$, perfect $\left.\left(s_{6}\right)\right\}$.

Table 3. The hesitant fuzzy linguistic term sets of alternatives.

\begin{tabular}{cccc}
\hline & $c_{1}$ & $c_{2}$ & $c_{3}$ \\
\hline$a_{1}$ & $\left\{s_{1}, s_{2}, s_{3}\right\}$ & $\left\{s_{2}, s_{3}\right\}$ & $\left\{s_{1}, s_{2}, s_{3}\right\}$ \\
\hline$a_{2}$ & $\left\{s_{1}, s_{2}, s_{3}\right\}$ & $\left\{s_{1}, s_{2}, s_{3}\right\}$ & $\left\{s_{-2}, s_{-1}, s_{0}\right\}$ \\
\hline$a_{3}$ & $\left\{s_{2}, s_{3}\right\}$ & $\left\{s_{1}, s_{2}, s_{3}\right\}$ & $\left\{s_{3}\right\}$ \\
\hline
\end{tabular}

By using the linguistic intuitionistic fuzzy set TOPSIS method (Algorithm 1), the LMCDM problem can be carried out as follows:

Step 1: According to Table 3 and Eq.(5), we use $S^{\prime}$ to obtain the linguistic intuitionistic fuzzy decision matrix $D$, i.e.,

$$
D=\begin{gathered}
c_{1} \\
a_{1} \\
a_{2} \\
a_{3}
\end{gathered}\left(\begin{array}{ccc}
\left(s_{4}, s_{0}\right) & \left(s_{5}, s_{0}\right) & c_{3} \\
\left(s_{4}, s_{0}\right) \\
\left(s_{4}\right) & \left(s_{4}, s_{0}\right) & \left(s_{1}, s_{3}\right) \\
\left(s_{5}, s_{0}\right) & \left(s_{4}, s_{0}\right) & \left(s_{3}, s_{3}\right)
\end{array}\right)
$$

In Table 3, such as for hesitant fuzzy linguistic term sets $\left\{s_{1}, s_{2}, s_{3}\right\}$ of $a_{1}$ with respect to $c_{1}$, in $S^{\prime}$, $\left\{s_{1}, s_{2}, s_{3}\right\}$ is transformed as $\left\{s_{4}, s_{5}, s_{6}\right\}$, then we select $s_{4}=\min \left\{s_{4}, s_{5}, s_{6}\right\}$ as the membership fuzzy linguistic assessment of $a_{1}$ with respect to $c_{1}$ and $s_{0}=$ $s_{g-\max }\{4,5,6\}$ as the nonmembership fuzzy linguistic assessment of $a_{1}$ with respect to $c_{1}$, i.e., the linguistic intuitionistic fuzzy set of $a_{1}$ with respect to $c_{1}$ is $\left(s_{4}, s_{0}\right)$, others linguistic intuitionistic fuzzy sets in $D$ can be similarly obtained. Formally, for any hesitant fuzzy linguistic term set $\left\{s_{i}, \ldots, s_{j}\right\}(i \leqslant \ldots \leqslant j)$ on $S=\left\{s_{0}, \ldots, s_{g}\right\},\left(s_{i}, s_{g-j}\right)$ is a linguistic intuitionistic fuzzy set on $S$ due to $i+g-j=g-(j-i) \leqslant$ $g$. This does not mean that linguistic intuitionistic fuzzy sets and hesitant fuzzy linguistic term sets on $S$ are equal to each other, because in real world practice, a decision maker provides a hesitant fuzzy linguistic term set $\left\{s_{i}, \ldots, s_{j}\right\}$, it does not imply that $s_{i}$ is membership fuzzy linguistic assessment and $s_{g-j}$ is nonmembership fuzzy linguistic assessment. Here, we only limit discussion in a formal form, and we think that hesitant fuzzy linguistic term set and linguistic intuitionistic fuzzy set are two alternative tools for representing linguistic assessments of alternatives in linguistic decision making. 
Step 2: For each column of $D$, we have

$$
\begin{aligned}
& \vee c_{1}=\left(\max \left\{s_{4}, s_{4}, s_{5}\right\}, \min \left\{s_{0}, s_{0}, s_{0}\right\}\right)=\left(s_{5}, s_{0}\right), \\
& \vee c_{2}=\left(\max \left\{s_{5}, s_{4}, s_{4}\right\}, \min \left\{s_{0}, s_{0}, s_{0}\right\}\right)=\left(s_{5}, s_{0}\right), \\
& \vee c_{3}=\left(\max \left\{s_{4}, s_{1}, s_{3}\right\}, \min \left\{s_{0}, s_{3}, s_{3}\right\}\right)=\left(s_{4}, s_{0}\right), \\
& \wedge c_{1}=\left(\min \left\{s_{4}, s_{4}, s_{5}\right\}, \max \left\{s_{0}, s_{0}, s_{0}\right\}\right)=\left(s_{4}, s_{0}\right), \\
& \wedge c_{2}=\left(\min \left\{s_{5}, s_{4}, s_{4}\right\}, \max \left\{s_{0}, s_{0}, s_{0}\right\}\right)=\left(s_{4}, s_{0}\right), \\
& \wedge c_{3}=\left(\min \left\{s_{4}, s_{1}, s_{3}\right\}, \max \left\{s_{0}, s_{3}, s_{3}\right\}\right)=\left(s_{1}, s_{3}\right) .
\end{aligned}
$$

Based on Eqs.(6) and (7), we obtain the positive and negative ideal solutions are

$$
\begin{aligned}
& \mathrm{PIS}=\operatorname{LIFWA}\left(\vee c_{1}, \vee c_{2}, \vee c_{3}\right)
\end{aligned}
$$

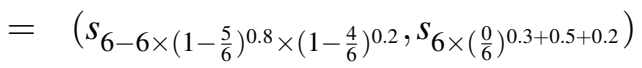

$$
\begin{aligned}
& \doteq\left(s_{4.86}, s_{0}\right) \text {, } \\
& \begin{aligned}
\mathrm{NIS} & =\left(s_{\alpha_{n}}, s_{\beta_{n}}\right)=\operatorname{LIFWA}\left(\wedge c_{1}, \wedge c_{2}, \wedge c_{3}\right) \\
& =\left(s_{\left.6-6 \times\left(1-\frac{4}{6}\right)^{0.8} \times\left(1-\frac{1}{6}\right)^{0.2}, s_{6 \times\left(\frac{0}{6}\right)^{0.8} \times\left(\frac{3}{6}\right)^{0.2}}\right)}\right. \\
& \doteq\left(s_{3.6}, s_{0}\right) .
\end{aligned}
\end{aligned}
$$

Step 3: For each row of $D$, according to Eq.(8), we have

$$
\begin{aligned}
& A_{1}=\operatorname{LIFWA}\left(\left(s_{4}, s_{0}\right),\left(s_{5}, s_{0}\right),\left(s_{4}, s_{0}\right)\right) \\
& =\left(s_{\left.\left.6-6 \times\left(1-\frac{4}{6}\right)^{0.5} \times\left(1-\frac{5}{6}\right)^{0.5}, s_{6 \times\left(\frac{0}{6}\right.}\right)^{0.3+0.5+0.2}\right)}\right. \\
& \doteq\left(s_{4.58}, s_{0}\right) \text {, } \\
& A_{2}=\operatorname{LIFWA}\left(\left(s_{4}, s_{0}\right),\left(s_{4}, s_{0}\right),\left(s_{1}, s_{3}\right)\right) \\
& \doteq\left(s_{3.6}, s_{0}\right) \text {, } \\
& A_{3}=\operatorname{LIFWA}\left(\left(s_{5}, s_{0}\right),\left(s_{4}, s_{0}\right),\left(s_{3}, s_{3}\right)\right) \\
& \doteq\left(s_{4.24}, s_{0}\right) \text {, }
\end{aligned}
$$

Step 4: For each alternative, according to Eqs.(9) and (10), we obtain the following Hamming distances

$$
\begin{aligned}
d\left(A_{1}, \mathrm{PIS}\right) & =\frac{|4.58-4.86|+|0-0|+|1.42-1.14|}{2} \\
& =0.28 \\
d\left(A_{1}, \mathrm{NIS}\right) & =\frac{|4.58-3.6|+|0-0|+|1.42-2.4|}{2} \\
& =0.98 \\
d\left(A_{2}, \mathrm{PIS}\right) & =1.26, d\left(A_{2}, \mathrm{NIS}\right)=0 \\
d\left(A_{3}, \mathrm{PIS}\right) & =0.62, d\left(A_{2}, \mathrm{NIS}\right)=0.64
\end{aligned}
$$

Based on Eqs.(11), (12) and (13), the maximum Hamming distance, the minimum Hamming distance and the relative closeness degree of each alternative are as follows:

$$
\begin{aligned}
d_{\text {max }}^{-} & =\max \{0.98,0,0.64\}=0.98 \\
d_{\text {min }}^{+} & =\min \{0.28,1.26,0.618\}=0.28 \\
C\left(x_{1}\right) & =\frac{1}{2}\left(\frac{0.98}{0.98}+\frac{0.28}{0.28}\right)=1 \\
C\left(x_{2}\right) & =\frac{1}{2}\left(\frac{0}{0.98}+\frac{0.28}{1.26}\right) \doteq 0.22 \\
C\left(x_{3}\right) & =\frac{1}{2}\left(\frac{0.64}{0.98}+\frac{0.28}{0.62}\right) \doteq 0.55
\end{aligned}
$$

Accordingly, the ranking of three alternatives is $a_{2} \prec$ $a_{3} \prec a_{1}$ according to Eq.(14), and the set of most satisfying alternatives is $A_{M S}=\left\{a_{1}\right\}$.

In the following, we compare the linguistic intuitionistic fuzzy set TOPSIS method with the HFLVIKOR method, the symbolic aggregation-based method and the HFL-TOPSIS method, in which, the HFL-VIKOR method, the symbolic aggregationbased method and the HFL-TOPSIS method transform assessments of alternatives into HFLTSs based on Table 3, however, the linguistic intuitionistic fuzzy set TOPSIS method transforms assessments of alternatives into linguistic intuitionistic fuzzy sets, we carry out comparison of four methods as follows.

a) The positive and negative ideal solutions: The HFL-VIKOR method ${ }^{46}$ and the HFL-TOPSIS method $^{48}$ utilized the score function, the variance function, the Max and Min operators of HFLTSs to obtain PIS and NIS, this means that their PIS or NIS are one of HFLTSs of alternatives, such as in Table $4,\left\{s_{3}\right\}$ of PIS is HFLTS of $c_{3},\left\{s_{-2}, s_{-1}, s_{0}\right\}$ of NIS is HFLTS of $c_{3}$. Its drawback is that PIS or NIS may be a HFLTS of an alternative, such as NIS $\left(\left\{s_{1}, s_{2}, s_{3}\right\},\left\{s_{1}, s_{2}, s_{3}\right\},\left\{s_{-2}, s_{-1}, s_{0}\right\}\right)$ is the HFLTS of $a_{2}$, i.e., this makes that $a_{2}$ is the worst alternative.

The symbolic aggregation-based method ${ }^{47}$ utilized Min-upper and Max-lower operators to obtain the upper bound and the lower bound of each HFLTS and construct the core information of alternatives (CIA), such as for $c_{1}$, Min bounds of $a_{1}, a_{2}$ and $a_{3}$ are $s_{1}, s_{1}$ and $s_{2}$, so the Min-upper of $c_{1}$ is $s_{2}$. Max bounds of $a_{1}, a_{2}$ and $a_{3}$ are $s_{3}, s_{3}$ and $s_{3}$, so the Max-lower of $c_{1}$ is $s_{3}$, hence CIA for $c_{1}$ is $\left[s_{2}, s_{3}\right]$. 
Intuitively, CIA reduces HFLTSs into linguistic intervals, especially, if the Min-upper is equal to the Max-lower for each criterion, then CIA contain certain linguistic information, such as $\left[s_{3}, s_{3}\right]$ for $c_{3}$ in Table 4, which maybe loss a lot of useful information, and there is no any hesitant fuzzy linguistic information in CIA.

In the linguistic intuitionistic fuzzy set TOPSIS method, we use linguistic intuitionistic fuzzy numbers on the linguistic term set $S$ to represent assessments of alternatives, and utilize Union and Intersection of linguistic intuitionistic fuzzy numbers and the linguistic intuitionistic fuzzy weighted averaging operator to obtain PIS and NIS, which are still linguistic intuitionistic fuzzy numbers, i.e., hesitant fuzzy linguistic information PIS are contained in PIS and NIS, because we use the linguistic intuitionistic fuzzy weighted averaging operator, our PIS and NIS are different with PIS and NIS in methods ${ }^{46,47,48}$, this can be seen from Table 4 .

b) The ranking of alternatives: In the HFLVIKOR method ${ }^{46}$, the hesitant fuzzy linguistic group utility measure $\mathrm{HFLGU}_{i}$ and the hesitant fuzzy individual regret measure $\mathrm{HFLIR}_{i}$ for the alternative $a_{i}$ are defined by the hesitant fuzzy linguistic Euclidean $L_{p}$-metric, then the hesitant fuzzy linguistic compromise measure $\mathrm{HFLC}_{i}$ is established,

$$
\begin{aligned}
\mathrm{HFLC}_{i}= & \theta \frac{\mathrm{HFLGU}_{i}-\mathrm{HFLGU}^{+}}{\mathrm{HFLGU}^{-}-\mathrm{HFLGU}^{+}}+ \\
& (1-\theta) \frac{\mathrm{HFLIR}_{1}-\mathrm{HFLIR}^{+}}{\mathrm{HFLIR}^{-}-\mathrm{HFLIR}^{+}}
\end{aligned}
$$

in which, $\mathrm{HFLGU}^{+}=\min \left\{\mathrm{HFLGU}_{1}, \mathrm{HFLGU}_{2}\right.$, $\left.\mathrm{HFLGU}_{3}\right\}, \mathrm{HFLGU}^{-}=\max \left\{\mathrm{HFLGU}_{1}, \mathrm{HFLGU}_{2}\right.$, $\left.\mathrm{HFLGU}_{3}\right\}, \mathrm{HFLIR}^{+}=\min \left\{\mathrm{HFLIR}_{1}, \mathrm{HFLIR}_{2}\right.$, $\left.\mathrm{HFLIR}_{3}\right\}$ and HFLIR ${ }^{-}=\max \left\{\mathrm{HFLIR}_{1}, \mathrm{HFLIR}_{2}\right.$, $\left.\mathrm{HFLIR}_{3}\right\}$, and $\theta \in[0,1]$ is the weight of the strategy of the majority of criteria or the maximum overall utility. By ranking $\mathrm{HFLGU}_{i}, \mathrm{HFLIR}_{i}$ and $\mathrm{HFLC}_{i}$ in descending order, the final optimal solution should be the one that makes those measures attain the minimum values. Formally, the HFL-VIKOR method is very effective in handling the noncommensurable criteria, derives the compromise solution(s) which consider not only maximizing the group utility for the majority but mini- mizing individual regret for the opponent as well and takes different weights of the criteria into account.

In the symbolic aggregation-based method ${ }^{47}$, based on the CIA of each alternative, a binary preference relation between two alternatives is calculated, and the nondominance degree (NDD) of each alternative is used to obtain the set of nondominated alternatives, which indicates the degree to which alternative $a_{i}$ is not dominated by the remaining ones. In the HFL-TOPSIS method ${ }^{48}$, the Euclidean distance measure is used to obtain distances between alternatives and the positive and negative ideal solutions, then the relative closeness degree (RC) of each alternative is calculated to rank alternatives, where weights of criteria are not used in the symbolic aggregation-based method and the HFLTOPSIS method.

In the linguistic intuitionistic fuzzy set TOPSIS method, we use Hamming distances between alternatives and the positive and negative ideal solutions to calculate the relative closeness of each alternative. As shown in Table 5, the ranking of alternatives are the same in the linguistic intuitionistic fuzzy set TOPSIS method and the HFL-VIKOR method $^{46}$, however, calculation of the linguistic intuitionistic fuzzy set TOPSIS method are simpler than the HFL-VIKOR method. We notice that the ranking results of the symbolic aggregation-based method and the HFL-TOPSIS method are different from that produced by the linguistic intuitionistic fuzzy set TOPSIS method and the HFL-VIKOR method, The reasons leading to this unconvincing result can be set out as follows: for one thing, with the symbolic aggregation-based method and the HFL-TOPSIS method, the weights of different criterion do not take into consideration; For another thing, when aggregating the HFLTSs by the symbolic aggregation operators, including the Min-upper operator and the Max-lower operator, the HFLTSs are reduced into linguistic intervals, which losses quite a lot of useful information. When aggregating the HFLTSs by Euclidean distance measure, including the normalized hesitant fuzzy linguistic terms, it only considers the distances from the ideal solution and from the negative-ideal solution, without considering their relative importance. 
Table 4: Main results of four methods

\begin{tabular}{|l|l|l|l|}
\hline & PIS & NIS & CIA \\
\hline The method $^{46}$ & $\left(\left\{s_{2}, s_{3}\right\},\left\{s_{2}, s_{3}\right\},\left\{s_{3}\right\}\right)$ & $\left(\left\{s_{1}, s_{2}, s_{3}\right\},\left\{s_{1}, s_{2}, s_{3}\right\},\left\{s_{-2}, s_{-1}, s_{0}\right\}\right)$ & - \\
\hline The method $^{47}$ & - & - & $\left(\left[s_{2}, s_{3}\right],\left[s_{0}, s_{1}\right],\left[s_{3}, s_{3}\right]\right)$ \\
\hline The method $^{48}$ & $\left(\left\{s_{2}, s_{3}\right\},\left\{s_{2}, s_{3}\right\},\left\{s_{3}\right\}\right)$ & $\left(\left\{s_{1}, s_{2}, s_{3}\right\},\left\{s_{1}, s_{2}, s_{3}\right\},\left\{s_{-2}, s_{-1}, s_{0}\right\}\right)$ & - \\
\hline Our method & $\left(s_{4.86}, s_{0}\right)$ & $\left(s_{3.6}, s_{0}\right)$ & - \\
\hline
\end{tabular}

Table 5: The ranking of four methods

\begin{tabular}{|c|c|c|c|c|}
\hline & Using wights & HFLC (NDDs or RC) & The ranking & The best one \\
\hline The method ${ }^{46}$ & $\sqrt{ }$ & $\left(0^{*}, 1^{-}, 0.6074\right)$ & $a_{2} \prec a_{3} \prec a_{1}$ & $a_{1}$ \\
\hline The method ${ }^{47}$ & - & $(0,0.5,1)$ & $a_{1} \prec a_{2} \prec a_{3}$ & $a_{3}$ \\
\hline The method ${ }^{48}$ & - & $(0.6531,0,0.8799)$ & $a_{2} \prec a_{1} \prec a_{3}$ & $\overline{a_{3}}$ \\
\hline Our method & $\sqrt{ }$ & $(1,0.22,0.55)$ & $a_{2} \prec a_{3} \prec a_{1}$ & $a_{1}$ \\
\hline
\end{tabular}

Summary, in Example 6, we respectively use hesitant fuzzy linguistic term sets and linguistic intuitionistic fuzzy sets to represent linguistic assessments of the LMCDM problem, then we compare the linguistic intuitionistic fuzzy set TOPSIS method with the HFL-VIKOR method, the symbolic aggregation-based method and the HFL-TOPSIS method, which are based on hesitant fuzzy linguistic term sets. An intriguing problem is which method is more reasonable among the above mentioned four methods or which representation of the above mentioned four methods is more reasonable? In real world practice, it is not possible to determine which one is the best suitable alternative for a given decision problem, this means that it is difficult to answer the intriguing problem. Theoretically, the testing criteria to evaluate the validity of MCDM methods in the same numerical data has been established such as in ${ }^{49}$, which can help us for our future research works.

\section{Conclusions}

Motivated by linguistic intuitionistic fuzzy numbers, in the paper, uncertain assessments information in linguistic multi-criteria decision makings are express by linguistic intuitionistic fuzzy sets on linguistic terms set, then Hamming distance between two linguistic intuitionistic fuzzy sets and their properties are presented and analyzed. Accordingly, the linguistic intuitionistic fuzzy set TOPSIS method for LMCDM problems is proposed, compared with the traditional TOPSIS methods, different the positive ideal solution, the negative ideal solution and the relative closeness degrees of alternatives are provided, based on the designed algorithm, LMCDM problems with linguistic intuitionistic fuzzy sets can be automatically carried out. An example is also utilized to illustrate the performance, usefulness and effectiveness of the linguistic intuitionistic fuzzy set TOPSIS method, and compare the method with the HFL-VIKOR method, the symbolic aggregationbased method and the HFL-TOPSIS method.

\section{Acknowledgments}

This work has been partially supported by the National Natural Science Foundation of China (Grant No.61372187), the scientific and technological project of Sichuan Province (2016GZ0099) and the open research fund of key laboratory of intelligent network information processing, Xihua University (szjj2014-052, szjj2015-061).

\section{References}

1. T. Evangelos, multiple attribute decision making methods: a comparative study, (Kluwer Academic Publishers, Dordrecht, 2000). 
2. R.T. Clemen, Making Hard Decisions. An Introduction to Decision Analysis, (Duxbury Press, 1995).

3. J. Lu, G. Zhang, D. Ruan, and F. Wu, Multi-Objective Group Decision Making. Methods, Software and Applications with Fuzzy Set Techniques, (Imperial College Press, 2007).

4. L. Martinez, R.M. Rodriguez, and F. Herrera, The 2-tuple Linguistic Model. Computing with Words in Decision Making, (Springer International Publishing Switzerland, 2015).

5. C.L. Hwang and K. Yoon, Multiple Attribute Decision Making: Methods and Applications, (Springer-Verlag, New York, 1981).

6. Z. Yue, TOPSIS-based group decision-making methodology in intuitionistic fuzzy setting, Information Sciences 277 (2014) 141-153.

7. F. Boran, S. Gene, M. Kurt, and D. Akay, A multicriteria intuitionistic fuzzy group decision making for supplier selection with TOPSIS method, Expert Systems with Applications 36 (2009) 11363-11368.

8. T.Y. Chen, Interval-valued fuzzy TOPSIS method with leniency reduction and an experimental analysis, Applied Soft Computing 11 (8) (2011) 4591-4606.

9. J.H. Park, I.Y. Park, Y.C. Kwun, and X.G. Tan, Extension of the TOPSIS method for decision making problems under interval-valued intuitionistic fuzzy environment, Applied Mathematical Modelling 35 (2011) 2544-2556.

10. E. Roszkowska and T. Wachowicz, Application of fuzzy TOPSIS to scoring the negotiation offers in illstructured negotiation problems, European Journal of Operational Research 242 (2015) 920-932.

11. H. Shih, H. Shyur, and E. Lee, An extension of TOPSIS for group decision making, Mathematical and Computer Modelling 45 (2007) 801-813.

12. Z. Yue, A method for group decision-making based on determining weights of decision makers using TOPSIS, Applied Mathematical Modelling 35 (4) (2001) 1926-1936.

13. Z. Yue, An extended TOPSIS for determining weights of decision makers with interval numbers, Knowledge-Based Systems 24 (2011) 146-153.

14. C.T. Chen, Extensions of the TOPSIS for group decision-making under fuzzy environment, Fuzzy Sets and Systems 114 (2000) 1-9.

15. B. Ashtiani, F. Haghighirad, A. Makui, and G. Montazer, Extension of fuzzy TOPSIS method based on interval-valued fuzzy sets, Applied Soft Computing 9 (2) (2009) 457-461.

16. Y.Y. He and Z.W. Gong, Extension of TOPSIS for intuitionistic fuzzy multiple attribute decision making and experimental analysis, Advances in Information Sciences and Service Sciences 4 (19) (2012) 397-405.

17. S. Liu, F. Yu, W. Xu, and W. Zhang, New approach to MCDM under interval-valued intuitionistic fuzzy en- vironment, International Journal of Machine Learning and Cybernetics 4 (6) (2013) 671-678.

18. Z. Yue, Group decision making with multi-attribute interval data, Information Fusion 14 (2013) 551-561.

19. R.M. Rodríguez, A. Labella and L. Martínez, An Overview on Fuzzy Modelling of Complex Linguistic Preferences in Decision Making, International Journal of Computational Intelligence Systems 9 (1) (2016) 81-94.

20. R.M. Rodríguez, L. Martínez, and F. Herrera, A group decision making model dealing with comparative linguistic expressions based on hesitant fuzzy linguistic term sets, Information Sciences 241 (1) (2013) 28-42.

21. R.M. Rodríguez, L. Martínez, V. Torra, Z.S. Xu, and F. Herrera, Hesitant fuzzy sets: state of the art and future directions, International Journal of Intelligent Systems 29 (6) (2014) 495-524.

22. H.C. Liao and Z.S. Xu, Approaches to manage hesitant fuzzy linguistic information based on the cosine distance and similarity measures for HFLTSs and their application in qualitative decision making, Expert Systems with Applications 42 (2015) 5328-5336.

23. M. Zeleny, A concept of compromise solutions and the method of the displaced ideal, Computers and $\mathrm{Op}$ erations Research 1 (1974) 479-496.

24. N. Banaeian, H. Mobli, B. Fahimnia, I.E. Nielsen, and M. Omid, Green supplier selection using fuzzy group decision making methods: A case study from the agrfood industry, Computers and Operations Research (2016), https://doi.org/10.1016/j.cor.2016.02.015.

25. M. Kucukvar, S. Gumus, G. Egilmez, and O. Tatari, Ranking the sustainability performance of pavements: An intuitionistic fuzzy decision making method, $A u$ tomation in Construction 40 (2014) 33-43.

26. H.S. Lee, Optimal consensus of fuzzy opinions under group decision making environment, Fuzzy Sets and Systems 132 (2002) 303-315.

27. E. Szmidt and J. Kacprzyk, Distances between intuitionistic fuzzy sets, Fuzzy Sets and Systems 114 (2000) 505-518.

28. Z. Yue, An avoiding information loss approach to group decision making, Applied Mathematical Modelling 37 (2013) 112-126.

29. H.M. Hsu and C.T. Chen, Aggregation of fuzzy opinions under group decision making, Fuzzy Sets and Systems 79 (3) (1996) 279-285.

30. F. Herrera and L. Martinez, A 2-tuple fuzzy linguistic representation model for computing with words, IEEE Transactions on Fuzzy Systems 8 (6) (2000) 746-752.

31. Z.S. Xu, A method based on linguistic aggregation operators for group decision making with linguistic preference relations, Information Sciences 166 (2004) 1930.

32. J.H. Wang and J. Hao, A new version of 2-tuple fuzzy linguistic representation model for computing with 
words, IEEE Transactions on Fuzzy Systems 14 (3) (2006) 435-445.

33. W.T. Guo, V.N. Huynh, and Y. Nakamori, A proportional 3-tuple fuzzy linguistic representation model for screening new product projects, Journal of Systems Science and Systems Engineering, 25 (1) (2016) 1-22.

34. Y. Dong, Y. Xu, and S. Yu, Computing the numerical scale of the linguistic term set for the 2-tuple fuzzy linguistic representation model, IEEE Transactions on Fuzzy Systems 17 (6) (2009) 1366-1378.

35. D.F. Li, Multiattribute group decision making method using extended linguistic variables, International Journal of Uncertainty, Fuzziness and KnowledgeBased Systems 17 (6) (2009) 793-806.

36. W.E. Yang, X.F. Wang, and J.Q. Wang, Counted linguistic variable in decision-making, International Journal of Fuzzy Systems 16 (2) (2014) 196-203.

37. G.W. Wei, Extension of TOPSIS method for 2-tuple linguistic multiple attribute group decision making with incomplete weight information, Knowledge and Information Systems 25 (2010) 623-634.

38. E. Cables, M.Socorro Garcia-Cascales and M. Teresa Lamata, The LTOPSIS: An alternative to TOPSIS decision-making approach for linguistic variables, $E x$ pert Systems with Applications 39 (2012) 2119-2126.

39. L. Martinez and F. Herrera, An overview on the 2tuple linguistic model for computing with words in decision making: Extensions, applications and challenges, Information Sciences 207 (1) (2012) 1-18.

40. W. Yang, Induced choquet integrals of 2-tuple linguistic information, International Journal of Uncertainty, Fuzziness and Knowledge-Based Systems 21 (2) (2013) 175-200.

41. S.P. Wan, Some hybrid geometric aggregation operators with 2-tuple linguistic information and their ap- plications to multi-attribute group decision making, International Journal of Computational Intelligence Systems 6 (4) (2013) 750-763.

42. Q. Pang, H. Wang and Z.H. Xu, Probabilistic linguistic term sets in multi-attribute group decision making, Information Sciences (2016), doi: 10.1016/j.ins.2016.06.021.

43. G. Wei and X. Zhao, Some dependent aggregation operators with 2-tuple linguistic information and their application to multiple attribute group decision making, Expert Systems with Applications 39 (5) (2012) 5881-5886.

44. Y.J. Xu, J.M. Merigo and H.M. Wang, Linguistic power aggregation operators and their application to multiple attribute group decision making, Applied Mathematical Modelling 36 (2012) 5427-5444.

45. Z.C. Chen, P.H. Liu, and Z. Pei, An approach to multiple attribute group decision making based on linguistic intuitionistic fuzzy numbers, International Journal of Computational Intelligence Systems 8 (4) (2015) 747-760.

46. H.C. Liao, Z.S. Xu, and X.J. Zeng, Hesitant fuzzy linguistic VIKOR method and its application in qualitative multiple crideria decision making, IEEE Transactions on Fuzzy Systems 23 (5) (2015) 1343-1355.

47. R.M. Rodríguez, L. Martínez, and F. Herrera, Hesitant fuzzy linguistic term sets for decision making, IEEE Transactions on Fuzzy Systems 20 (1) (2012) 109-119.

48. I. Beg and T. Rashid, TOPSIS for hesitant fuzzy linguistic term sets, International Journal of Intelligent Systems 28 (2013) 1162-1171.

49. X. Wang and E. Triantaphyllou, Ranking irregularities when evaluating alternatives by using some ELECTRE methods, Omega 36 (2008) 45-63. 